\title{
WASHINGTON STATE UNIVERSITY NATURAL RADIOCARBON MEASUREMENTS II
}

\section{J G SHEPPARD and R M CHATTERS}

Department of Chemical and Nuclear Engineering, College of Engineering, Washington State University, Pullman, Washington 99163

This list of radiocarbon age measurements is for the period 19721974. Most of the samples are of archaeologic interest. The pretreatments of samples used are basically those described by Ralph and Michael (1971). All samples were converted to methane using the method developed by Fairhall, Schell, and Takashima (1961) and counted in $.5 \mathrm{~L}$ counting tubes at 2 to $4 \mathrm{~atm}$ of pressure. The WSU Radiocarbon Laboratory has been relocated to the basement of Dana Hall (three stories) and additional shielding has been added. The backgrounds of the counting tubes are .90 counts $/ \mathrm{min}$ and have an atmospheric pressure dependence of -.035 counts $/ \mathrm{min} / \mathrm{cm} \mathrm{Hg}$.

All ages were calculated using a ${ }^{14} \mathrm{C}$ half-life of 5568 years and NBS oxalic acid as contemporary reference material. Nineteenth century oak obtained from $\mathrm{E}$ Ralph was used as a secondary standard. The standard deviations reported represent counting errors of the sample, oxalate standard, and backgrounds. Corrections for isotopic fractionation or long-term variations of the ${ }^{14} \mathrm{C}$ production rate (Damon et al, 1972) were not made.

Samples WSU-1259-1328 were processed under the direction of R M Chatters, who retired in 1972. The remainder were processed under the direction of J C Sheppard. Samples were processed by Y Welter. M A Gass prepared much of the manuscript.

\section{SAMPLE DESCRIPTIONS \\ I. ARCHAEOLOGIC SAMPLES}

\section{W asden site series, Idaho}

The Wasden site is a large, loess-filled volcanic blister $29 \mathrm{~km} \mathrm{~W}$ of Idaho Falls, Idaho, at the foot of Kettle Butte $\left(43^{\circ} \mathrm{N}, 112^{\circ} 23^{\prime} \mathrm{W}\right)$ and contains a long sequence of faunal and cultural remains (Butler, 1972). Fragments of elephant (genus and species unkown) bone were recovered between 4.8 and $5.3 \mathrm{~m}$ (bedrock) below surface of fill. The upper elephant bone material consisted of naturally weathered neural arch fragments, while that resting on the bedrock floor of the cave consisted mainly of a scapula lacking the articular end and of cracked, broken, partially roasted and charred long bones.

\section{WSU-1259. Owl Cave, Snake River Plain, $\quad 12,250 \pm 200$ Idaho $10,300 \mathrm{BC}$}

Neural arch fragments from ca $5 \mathrm{~cm}$ below surface of fill. Coll 1971 by members of the Upper Snake River Prehistoric Soc, Inc; subm 1971 by B R Butler, Idaho State Univ Mus, Pocatello, Idaho. 


\section{WSU-1281. Owl Cave, Idaho}

Charred long bone resting on bedrock $5.25 \mathrm{~cm}$ below surface. Coll 1971 by B R Butler and members of the Upper Snake River Soc, Inc; subm 1971 by B R Butler.

\section{Redfish Lake Overhang series, Idaho}

Redfish Lake Overhang is located $\mathrm{N}$ of Little Redfish Lake, Custer Co, Idaho $\left(44^{\circ} 10^{\prime} \mathrm{N}, 114^{\circ} 54^{\prime} \mathrm{W}\right)$.

\section{WSU-1395. Redfish Lake, Idaho}

$9860 \pm 180$ 7910 BC

Charcoal sample from hearth 95 to $100 \mathrm{~cm}$ below surface and at interface of sandy loam and hard-packed layer. Sample was from a cache of 9 point blanks, 1 semi-finished projectile point (tentatively id as "Haskett-Lake Mojave") and 3 scrapers. Coll 1972 by K Sargeant; subm 1972 by K Sargeant and B R Butler.

\section{WSU-1396. Redfish Lake, Idaho}

$10,500 \pm 180$ 8550 BC

Charcoal from a possible fireplace, 80 to $105 \mathrm{~cm}$ below surface, and at extreme rear of cave. Sample was enclosed in coarse sandy loam mixed with cobbles and boulders redeposited from a glacial moraine. Coll 1972 by K Sargeant; subm 1972 by K Sargeant and B R Butler.

\section{WSU-1397. Redfish Lake, Idaho}

$8060 \pm 190$

Charcoal from organic stratum 69 to $77 \mathrm{~cm}$ below surface and $170 \mathrm{~cm}$ N and $130 \mathrm{~cm} \mathrm{~W} \mathrm{of} \mathrm{WSU-1396.} \mathrm{Coll} 1972$ by M Dahlstrom; subm 1972 by K Sargeant and B R Butler.

\section{WSU-1410. Redfish Lake, Idaho}

$$
670 \pm 130
$$

\section{AD 1280}

Charcoal from coarse sandy stratum underlying glacial till near WSU-1397 and $120 \mathrm{~cm}$ below surface of WSU-1395. Coll and subm 1972 by $\mathrm{K}$ Sargeant and B R Butler.

\section{WSU-1423. American Falls Reservoir, Idaho}

$21,500 \pm 700$

$$
19,550 \mathrm{BC}
$$

Bone from Bronco Jim Point, Pierce Co, Idaho $\left(42^{\circ} 56^{\prime} \mathrm{N}, 112^{\circ}\right.$ $41^{\prime} \mathrm{W}$ ), from sand and gravel layer 1.5 to $1.8 \mathrm{~m}$ below surface. Fauna from this layer includes horse, camel, bison, ground sloth, mammoth, bear, dire wolf, and saber-tooth cat. Coll 1973 by H Emry; subm 1973 by B R Butler.

\section{WSU-1424. American Falls Reservoir, Idaho $\mathbf{2 9 , 3 5 0}$ BC}

Bone fragments from Rainbow Beach, Power Co, Idaho $\left(42^{\circ} 53^{\prime} \mathrm{N}\right.$, $112^{\circ} 43^{\prime} \mathrm{W}$ ), from sand and gravel layer 1.5 to $1.8 \mathrm{~m}$ below surface. Fauna from this layer includes horse, camel, bison, ground sloth, mammoth, bear, dire wolf, and saber-tooth cat. Coll 1973 by G McDonald; subm 1973 by B R Butler. 


\section{WSU-1441. Blackfoot Reservoir, Idaho}

Charcoal from campsite, Feature 3, Site 10-BM-50 (Poison Creek), on a tributary of Blackfoot $\mathrm{R}\left(111^{\circ} 40^{\prime} \mathrm{N}, 43^{\circ} 0^{\prime} \mathrm{W}\right)$. Coll and subm 1973 by B R Butler. Comment (BRB): sample coll during 1973 test excavations of Blackfoot Reservoir Project and came from hearth of well-defined pottery-bearing occupation that should date between AD 1450 and AD 1750, ie, after appearance of pottery in region but prior to introduction of the horse.

\section{WSU-1323. Kamiah, Idaho}

$1170 \pm 170$

Charcoal from hearth 50 to $60 \mathrm{~cm}$ below surface $14.5 \mathrm{~km}$ downriver from Kamiah. Dates upper limit for assoc with Cascade and Side-Notched points. Coll 1970 by V Platt; subm 1972 by Idaho State Univ, Pocatello, Idaho.

WSU-1324. Kamiah, Idaho

$4710 \pm 180$

$2760 \mathrm{BC}$

Charcoal from same trench as WSU-1323, 140 to $160 \mathrm{~cm}$ below surface. Dates lower limit for assoc of Cascade and Side-Notched points. Coll 1970 by V Platt; subm 1972 by Idaho State Univ.

\section{$3770 \pm 170$}

Charcoal from basal gravel of lower terrace of Big I., North Fork Clearwater R, Clearwater Co $\left(46^{\circ} 38^{\prime} \mathrm{N}, 116^{\circ} \mathrm{W}\right)$. Coll 1971 by $\mathrm{D}$ Corliss; subm 1971 by E Swanson, Idaho State Univ Mus, Pocatello, Idaho.$$
\text { WSU-1278. North Fork Clearwater River, Idaho } 170 \text { BC }
$$

Charcoal, $40 \mathrm{~cm}$ below surface of Big I. site, upper terrace. Coll 1971 by D Corliss; subm 1971 by E Swanson.

\section{WSU-1305. North Fork Clearwater River, Idaho AD 700}

Charcoal, 40 to $50 \mathrm{~cm}$ below surface of Big I. site, middle terrace. Sample with cobble tools. Coll 1971 by D Corliss; subm 1972 by E Swanson.

\section{WSU-1302. Lenore site, Idaho}

Charcoal from site $.65 \mathrm{~km} \mathrm{~W}$ of Lenore, Nez Perce Co, Idaho $\left(46^{\circ}\right.$ $\left.31^{\prime} \mathrm{N}, 116^{\circ} 35^{\prime} \mathrm{W}\right)$. Sample was $246 \mathrm{~cm}$ below surface, in House $\mathrm{D}$, assoc with fire-cracked rubble. Coll 1971 by $\mathrm{R}$ Warner; subm by $\mathrm{E}$ Swanson.

\section{WSU-1303. Lenore site, Idaho}

Charcoal taken from site $.65 \mathrm{~km} \mathrm{~W}$ of Lenore, Nez Perce Co $\left(46^{\circ}\right.$ $\left.31^{\prime} \mathrm{N}, 116^{\circ} 35^{\prime} \mathrm{W}\right)$. Sample was from uppermost fireplace in Floor I, House J. Coll 1967 by D Morse; subm 1971 by E Swanson. 
WSU-1408B. Antelope Overhang, Oregon

$7960 \pm 120$

$6010 \mathrm{BC}$

Pieces of twisted fiber from inner bark of an unknown tree or shrub used in construction of prehistoric sandals at a large overhang on Antelope Creek, tributary of S Fork of the Owyhee R, Malheur Co, Oregon, $56.4 \mathrm{~km} \mathrm{~S} \mathrm{SW}$ of Jordan Valley. Sample assoc with a sandal of the same material underlying what appeared to be modern horse or cattle dung. Coll and subm 1969 by B R Butler. This sample dates cordage from the Antelope Overhang analyzed and described by Adavasio (1970).

\section{WSU-1326. Hells Canyon, Oregon}

$940 \pm 150$

Bones of Ovis canadensis and Odocoilens hemious from Hells Canyon Creek rockshelter, $56.4 \mathrm{~km} \mathrm{SE}$ of Enterprise, Wallowa Co $\left(45^{\circ} 15^{\prime}\right.$ $\left.\mathrm{N}, 116^{\circ} 40^{\prime} \mathrm{W}\right)$. Sample dates rockfall believed to be of Gannett Peak stage (Richmond, 1965). Coll 1967 by J Bross and F Turner; subm 1972 by M G Pavesic, Idaho State Univ, Pocatello, Idaho.

\section{WSU-1327. Hells Canyon, Oregon}

$590 \pm 110$

Charcoal, $96 \mathrm{~cm}$ below surface of Hells Canyon Creek rockshelter. Coll 1967 by S Troutman and C Sims; subm 1972 by M G Pavesic.

\section{WSU-1328. Hells Canyon, Oregon}

$1240 \pm 140$

Bones of Ovis canadensis and Odocoilens hemious from Hells Canyon Creek rockshelter, $175 \mathrm{~cm}$ below surface. Coll 1967 by J Gross and J Todd; subm 1972 by M G Pavesic.

\section{WSU-1422. Lind Coulee, Washington}

$8600 \pm 65$

$6650 \mathrm{BC}$

Humus from Slot Trench 7 to $9 \mathrm{~cm}$ below datum of Lind Coulee site, 45-GR-97 $\left(46^{\circ} 09^{\prime} \mathrm{N}, 119^{\circ} 00^{\prime} \mathrm{W}\right)$. Date represents an "apparent mean residence time" of carbon in this soil layer (Scharpenseel, 1972). Coll and subm 1972 by R Fryxell.

\section{Asotin series, Washington}

Charcoal excavated from sites in Asotin Co, Washington $\left(46^{\circ} \mathrm{N}\right.$, $\left.117^{\circ} \mathrm{W}\right)$. Coll and subm 1973 by F C Leonhardy, Anthropol Dept, Washington State Univ, Pullman, Washington.

\section{WSU-1437. Asotin, Washington}

$1480 \pm 80$ AD 470

Sample 73L2 was charcoal presumably assoc with fill in House 3 at Site 45AS82, Asotin Co. Cultural material from house was same as that dated by WSU-1438; estimated date: 4000 to 5000 yr BP. Area was badly disturbed both in historic and prehistoric times. Charcoal was found adjacent to an intrusive pit. Sample age indicates it was assoc with a prehistoric intrusion and not with House 3. 


\section{WSU-1438. Asotin, Washington}

$4060 \pm 130$

Sample 73L1 was charcoal from a geologic stratum designated informally "The Calcareous Sand" at Site 45AS82, Asotin Co. Based on geologic position and known age of underlying geologic strata and cultural materials, estimated age: between 4000 and 5000 yr BP. Sample provides a single date for an archaeologic assemblage not previously recognized as distinctive in Lower Snake River culture sequence (Leonhardy and Rice, 1970). Material probably represents a culture ancestral to that of the Tucannon phase, with a minimum initial date of 3000 вр and a terminal date of 2500 BP. Date of WSU-1438 is consistent with estimates based on both regional geologic sequence and regional cultural sequence.

WSU-1439. Asotin, Washington

$1410 \pm 80$

Sample 73L3 was charcoal from upper floor of House 2, Site 45AS82, Asotin Co. Estimated date: 2000 BP.

\section{WSU-1440. Asotin, Washington}

$$
1330 \pm 110
$$

Sample 73L4 was charcoal (burned grass and annual herbs) from lower floor of House 1, Site 45AS80, Asotin Co. Estimated date 2000 BP. Comment (FCL): WSU-1439 and -1440 date 2 different houses containing identical cultural materials. Dates confirm approx contemporaneity of houses and are somewhat later than estimated.

WSU-1443. Sekiu, W ashington

$2750 \pm 90$ 800 BC

Wood from Sq II, Level II, Site 45-CA-213, Sekiu, Clallam Co $\left(48^{\circ} 17^{\prime} \mathrm{N}, 124^{\circ} 21^{\prime} \mathrm{W}\right)$. Coll 1973 and subm 1974 by $\mathrm{D}$ Croes and R Daugherty, Anthropol Dept, Washington State Univ, Pullman, Wash.

\section{WSU-1442. Sekiu, Washington}

$2210 \pm 70$

Wooden fishhook from Sq I, Level III, Site 45-CA-213, $2.42 \mathrm{~km} \mathrm{~W}$ of Sekiu, Clallam Co (48 $\left.17^{\prime} \mathrm{N}, 124^{\circ} 21^{\prime} \mathrm{W}\right)$. Coll 1973 and subm 1974 by D Croes and R Daugherty.

\section{WSU-1318. Putu site, Alaska}

Charcoal from Putu site, Sagavanirktok Valley, Brooks Range, Alaska $\left(68^{\circ} 21^{\prime} \mathrm{N}, 148^{\circ} 50^{\prime} \mathrm{W}\right)$. Sample $.5 \mathrm{~m}$ below surface, $\mathrm{S} 7.3 \mathrm{~m}$, $\mathrm{W} .6 \mathrm{~m}$ from datum. The Putu site appears to be a single-component, short-term occupation. Artifacts from site include 4 fragments of fluted points, burins, blades, blade cores, large bi-faces, end-scraper on a blade, and utilized flakes. Occupation zone rests directly on glacial gravels covered by .3 to $.6 \mathrm{~m}$ light brown soil. Coll 1970 ; subm 1972 by $\mathrm{H} \mathrm{L}$ Alexander, Simon Fraser Univ, British Columbia. 
WSU-1420. Kettle Falls, Washington

$2960 \pm 60$

$1010 \mathrm{BC}$

Carbon from Stratum 5, Site 45-FE-45, Kettle Falls I., Columbia R

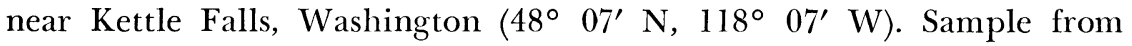
between $\mathrm{I}$ and $1.30 \mathrm{~m}$ below surface of midden. Site has been flooded by Lake Roosevelt since 1940. Coll 1972 and subm 1973 by D Chance and D Rice, Univ Idaho, Dept Anthropol, Moscow, Idaho.

\section{WSU-1421. Hanford, Washington}

$1860 \pm 60$

Shell (Margaritifera) from base of Stratum 3B at archeol Site 45BN-179 on S bank of Columbia R, USAEC Reservation, Hanford $\left(46^{\circ}\right.$ $39^{\prime} \mathrm{N}, 119^{\circ} 47^{\prime} \mathrm{W}$ ). Sample from midden at an alt 98.3 to $98.2 \mathrm{~m}$, grid coordinates $39-41 \mathrm{~N}, 74-76 \mathrm{E}$. Coll and subm 1973 by D Rice.

WSU-1456. Richland, Washington

Modern, pre-bomb

Charcoal and carbonized wood, from Site 45-BN-161, Sq V-19, Level II, 1.5 to $1.7 \mathrm{~m}$ below datum, at Columbia Park I., Richland $\left(46^{\circ} 38^{\prime} \mathrm{N}\right.$, $\left.119^{\circ} 45^{\prime} \mathrm{W}\right)$. Coll 1969; subm 1974 by D Rice.

\section{WSU-1457. Pasco, Washington}

AD 1500

$450 \pm 60$

Charcoal from a burned house plank from Site 45-FR-101, Sq U-33-S, Level 4, .5m below datum, at Chiawana Park, Pasco $\left(46^{\circ} 38^{\prime} \mathrm{N}, 119^{\circ}\right.$ 45’ W). Coll 1967 by Brooks; subm 1974 by D Rice.

\section{WSU-1458. Umatilla, Oregon}

$$
210 \pm 60
$$

AD 1740

Charcoal from Site 35-UM-35C/X-656-66, Sq 6F, 18-19, Level 10, 1.3 to $1.5 \mathrm{~m}$ below datum, Umatilla $\left(45^{\circ} 53^{\prime} \mathrm{N}, 119^{\circ} 20^{\prime} \mathrm{W}\right)$. Coll 1974 by V Eaton; subm 1974 by D Rice.

\section{WSU-1431. Last Supper Cave, Nevada}

$8630 \pm 195$

Shell from basal shell and stratum at Last Supper Cave, Site 26Hu-102, Humbolt Co, Nevada. Coll and subm 1973 by $\mathrm{T}$ N Layton, Nevada State Mus, Carson City, Nevada.

\section{WSU-1454. Gearhart, Oregon}

$$
860 \pm 100
$$

Charcoal from firepit $.75 \mathrm{~m}$ below surface of Site 35-CLT-8, on E side of sand dune ca $1.21 \mathrm{~km}$ from Pacific Ocean, Gearhart $\left(46^{\circ} 03^{\prime} 16^{\prime \prime}\right.$ $\mathrm{N}, 123^{\circ} 54^{\prime} 53^{\prime \prime} \mathrm{W}$ ). The site, left by Clatsop Indians (Lower Chinook), is composed of alternating layers of razor clam shells and sand. Coll 1973; subm 1974 by F Hasle, Clatsop Comm Coll, Astoria, Oregon.

\section{WSU-1455. Gearhart, Oregon}

$730 \pm 110$

Charcoal from firepit $1.95 \mathrm{~m}$ below surface of Site 35-CLT-8. Coll 1974 by Shrakey, Sanford, Lotterer, and Gillette; subm 1974 by F Hasle. 


\section{WSU-1429. Blue Lake, Idaho}

\section{AD 1240}

Charcoal, twigs, and wood fragments separated from gyttja at sediment depth 1.43 to $1.48 \mathrm{~m}$ of Blue Lake, Nez Perce Co, Idaho $\left(46^{\circ} 13^{\prime}\right.$ $\left.\mathrm{N}, 116^{\circ} 51^{\prime} \mathrm{W}\right)$. Coll and subm 1973 by $\mathrm{P} \mathrm{J}$ Meliringer and $\mathrm{K} \mathrm{L}$ Petersen, Washington State Univ Anthropol Dept, Pullman, Wash.

\section{WSU-1430. Blue Lake, Idaho}

$1340 \pm 80$

Charcoal, twigs, wood fragments and gyttja at sediment depth 2.42 to $2.47 \mathrm{~m}$ of Blue Lake, Nez Perce, Idaho $\left(46^{\circ} 13^{\prime} \mathrm{N}, 116^{\circ} 51^{\prime} \mathrm{W}\right)$. Coll and subm 1973 by P J Mehringer and K L Petersen.

\section{Wildcat Lake series, Washington}

Whole sediment core samples, primarily algal gyttja, from Wildcat

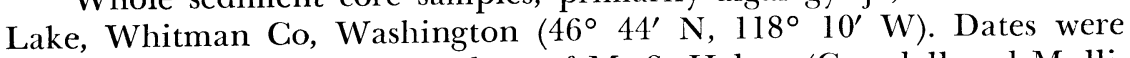
used to confirm tephra chronology of Mt St Helens (Crandall and Mullineaux, 1973) and Mt Mazama (Kittelman, 1973) eruptions and for fossil pollen studies. Coll 1972 by P J Mehringer and K L Petersen; subm by $\mathbf{P} \mathrm{J}$ Mehringer.

General Comment: 1445 SI and SII provide an upper limit radiocarbon age estimate for Mt St Helens "W" tephra layer at $1.50 \mathrm{~m}$. $1451 \mathrm{~S}$ and $\mathrm{H}$ provide an upper limit radiocarbon age for Mt St Helens "Y" tephra layer at $9 \mathrm{~m}$. WSU-1452 S is above a Mt Mazama tephra layer.

\begin{tabular}{|c|c|c|c|}
\hline Sample & Material & Depth & Age \\
\hline WSU-1445 SI & Sediment & $1.58-1.68 \mathrm{~m}$ & $\begin{array}{r}390 \pm 100 \\
\text { AD } 1560\end{array}$ \\
\hline WSU-1445 SII & Sediment & $1.58-1.68 \mathrm{~m}$ & $\begin{array}{l}400 \pm 60 \\
\mathrm{AD} 1550\end{array}$ \\
\hline WSU-1448 S & Sediment & $3.21-3.29 \mathrm{~m}$ & $\begin{array}{l}900 \pm 100 \\
\text { AD } 1050\end{array}$ \\
\hline WSU-1449 S & Sediment & $4.76-4.84 \mathrm{~m}$ & $\begin{array}{l}1140 \pm 100 \\
\operatorname{AD} 810\end{array}$ \\
\hline WSU-1446 & Sediment & $5.92-5.99 \mathrm{~m}$ & $\begin{array}{r}1800 \pm 100 \\
\text { AD } 150\end{array}$ \\
\hline WSU-1450 S & Sediment & $7.61-7.69 \mathrm{~m}$ & $\begin{array}{c}2300 \pm 100 \\
350 \mathrm{BC}\end{array}$ \\
\hline WSU-1451 H & Humic acid & $9.41-9.45 \mathrm{~m}$ & $\begin{array}{l}3690 \pm 90 \\
1740 \mathrm{BC}\end{array}$ \\
\hline WSU-1451 S & Sediment & $9.41-9.45 \mathrm{~m}$ & $\begin{array}{l}4180 \pm 90 \\
2230 \mathrm{BC}\end{array}$ \\
\hline WSU-1452 S & Sediment & $11.25-11.34 \mathrm{~m}$ & $\begin{array}{l}5380 \pm 130 \\
3430 \mathrm{BC}\end{array}$ \\
\hline
\end{tabular}




\section{Little Lake series, California}

Whole sediment core samples of Chara ooze or peat from Little Lake, California $\left(36^{\circ} 57^{\prime} \mathrm{N}, 117^{\circ} 54^{\prime} \mathrm{W}\right)$ to provide a chronology for fossil pollen and sediment studies. Coll 1974 by $\mathrm{P}$ J Mehringer and E L Davis; subm 1974 by P J Mehringer.

\begin{tabular}{llclr}
\hline Sample & Material & Depth & Age & $\%$ Modern \\
\hline WSU-1460 & Chara sp & & & $63.4 \pm 2.1$ \\
WSU-1473 & Potamogeton sp & & & $68.7 \pm 1.8$ \\
WSU-1461 & Chara ooze & $1.26-1.34 \mathrm{~m}$ & & $40.9 \pm 0.6$ \\
WSU-1490 & Carbonate & $1.26-1.34 \mathrm{~m}$ & & $40.3 \pm 0.6$ \\
WSU-1462 & Chara ooze & $3.58-3.48 \mathrm{~m}$ & & $47.2 \pm 1.0$ \\
WSU-1462H & Humic acid & $3.58-3.48 \mathrm{~m}$ & & $45.2 \pm 1.0$ \\
WSU-1465 & Chara ooze & $5.00-5.11 \mathrm{~m}$ & & \\
WSU-1474 & Peat & $5.91-6.01 \mathrm{~m}$ & $3020 \pm 120$ & \\
& & & $1070 \mathrm{BC}$ & \\
WSU-1466 & Peat & $7.96-8.06 \mathrm{~m}$ & $3920 \pm 120$ & \\
& & & $1970 \mathrm{BC}$ & \\
WSU-1463 & Chara ooze & $8.40-8.50 \mathrm{~m}$ & & $30.7 \pm 0.6$ \\
WSU-1464 & Peat & $11.30-11.37 \mathrm{~m}$ & $5060 \pm 140$ & \\
& & & $3110 \mathrm{BC}$ & \\
WSU-1464H & Humic acid & $11.30-11.37 \mathrm{~m}$ & $5000 \pm 140$ & \\
& & & $3050 \mathrm{BC}$ & \\
& & &
\end{tabular}

General Comment: (JCS \& PJM): ${ }^{14} \mathrm{C}$ ages for modern Potamogeton and Chara grown in Little Lake and the lake sediment derived from these aquatic plants were anomalously old. ${ }^{14} \mathrm{C}$ ages of peat samples are reasonable. Results are very similar to those observed by Deevey et al (1954) and can be explained on the basis that Chara and Potamogeton metabolized "old" $\mathrm{HCO}_{3}-$, while emergent aquatics (Typha and Scirpus), which produced the peat, metabolized atmospheric $\mathrm{CO}_{2}$. Calculations, based on peat data and assumption of a constant sedimentary deposition rate, indicate that the $\mathrm{HCO}_{3}-$ metabolized by the Chara and Potamogeton was initially $50 \%$ "old" carbon, suggesting reaction:

$$
\mathrm{CaCO}_{3}+{ }^{14} \mathrm{CO}_{2}+\mathrm{H}_{2} \mathrm{O}=\mathrm{Ca}^{2}++\mathrm{HCO}_{3}{ }^{-}+\mathrm{H}^{14} \mathrm{CO}_{3}-
$$

is important. Mixing of "old" spring water with atmospheric $\mathrm{CO}_{2}$ cannot be excluded. Analysis of ${ }^{14} \mathrm{C}$ content of the spring water, which feeds Little Lake, should clarify the situation.

\section{WATER SAMPLES}

WSU-1432. Giant Springs Modern

Water $\left(\mathrm{BaCO}_{3}\right)$ Sample 1 from spring near Missouri $\mathrm{R}$ at Great Falls, Madison I., Montana $\left(47^{\circ} 31^{\prime} \mathrm{N}, 111^{\circ} 15^{\prime} \mathrm{W}\right)$. Coll and subm 1973 by G Grimestad, Missoula, Montana. 
WSU-1433. General Mills Well

$66.3 \pm 2.6 \%$

Modern

Water $\left(\mathrm{BaCO}_{3}\right)$ Sample 2 from domestic well, Madison I. $\left(47^{\circ} 25^{\prime}\right.$ $\left.\mathrm{N}, 111^{\circ} 09^{\prime} \mathrm{W}\right)$. Coll and subm 1973 by $\mathrm{G}$ Grimestad.

\section{WSU-1435. J Bryant Well}

$42.6 \pm 1.1 \%$

Water $\left(\mathrm{BaCO}_{3}\right)$ Sample 4 from domestic well, Madison I. $\left(47^{\circ} 30^{\prime}\right.$ N, $\left.111^{\circ} 16^{\prime} \mathrm{W}\right)$. Coll and subm 1973 by G Grimestad.

\section{WSU-1436. Giant Springs}

$54.1 \pm 1.5 \%$

Modern

Water $\left(\mathrm{BaCO}_{3}\right)$ Sample 5. duplicate of WSU-1432, from spring near Missouri R in Great Falls, Madison I., Montana $\left(47^{\circ} 31^{\prime} \mathrm{N}, 111^{\circ} 15^{\prime}\right.$ W). Coll and subm 1973 by G Grimestad.

\section{MISCELLANEOUS SAMPLES}

\section{WSU-1428. Prudhoe Bay, Alaska}

Tamarack wood fragment from British Petroleum oil well at ca $122 \mathrm{~m}$ level, Prudhoe Bay, Alaska $\left(70^{\circ} 30^{\prime} \mathrm{N}, 148^{\circ} 30^{\prime} \mathrm{W}\right)$. Specimen represents underside of a branch, evidenced by compression wood, which is characteristic of branches and strongly leaning trees. Subm 1973 by F R Johnson, State Forester's Office, Alaska Dept Nat Resources, Anchorage, Alaska.

\section{WSU-1426. Prudhoe Bay, Alaska}

$$
25,500 \pm 1000
$$

Tamarack wood fragment from British Petroleum oil well at ca $122 \mathrm{~m}$ level, Prudhoe Bay, Alaska $\left(70^{\circ} 30^{\prime} \mathrm{N}, 148^{\circ} 30^{\prime} \mathrm{W}\right)$. Sample appears to be part of bole near ground line. Subm 1973 by F R Johnson.

\section{WSU-1453. Chewelah, Washington}

Log (Pinus Ponderosa) underlying bison skull excavated from bog at Chewelah, Washington $\left(48^{\circ} 15^{\prime} \mathrm{N}, 117^{\circ} 45^{\prime} \mathrm{W}\right)$. Coll and subm 1973 by C Gustafson, Anthropol Dept, Washington State Univ, Pullman, Wash.

\section{REFERENCES}

Adavasio, J M, 1970, The origin, development and distribution of Western archaic textiles: Tebiwa, v 12, no. 2, p 1-40.

Butler, B R, 1962, Contributions to the Prehistory of the Columbia Plateau: Idaho State Univ Mus occas papers, no. 9, Pocatello; and A Guide to Understanding Idaho Archaeology, 1968, 2nd ed, Idaho State Univ Mus, spec pub, Pocatello.

1972, The Holocene in the Desert West and its cultural significance, in: Fowler, D D (ed), Great Basin cultural ecology, a symposium: Desert Research Inst Pubs in Soc Sci, no. 8, p 5-12.

Crandell, D R and Mullineaux, D R, 1973, Pine Creek volcanic assemblage at $\mathrm{Mt}$ St Helens, Washington: US Geol Survey Bull 1383-A. 
Damon, P E, Long, A, and Wallick, E I, 1972, Dendrochronologic calibration of the carbon-14 time scale: 8th internatl conf on radiocarbon dating Proc, Lower Hutt, New Zealand, p 45-50.

Deevey, E S, Gross, M S, Hutchinson, G E, and Kraybill, H L, 1954, The natural $\mathrm{C}^{14}$ contents of materials from hard-water lakes: Nat Acad Sci Proc, v 40, p 285-288.

Fairhall, A W, Schell, W R, and Takashima, Y, 1961, Apparatus for methane synthesis for radiocarbon dating: Rev Sci Instruments, v 32, no. 3, p 323-325.

Kitteleman, L R, 1973, Mineralogy correlation and grain-size distributions of Mazama tephra and other postglacial pyroclastic layers, Pacific Northwest: Geol Soc America Bull, v 84, p 2957.

Leonhardy, F C and Rice, D G, 1970, A proposed culture typology for the Lower Snake River region, Southeastern Washington: Northwest Anthropol Research Notes, $\mathrm{v} 4$, no. 1.

Michael, H N and Ralph, E (eds), 1971, Climates, tree rings, and archaeology, in: Dating techniques for the archacologist, MIT Press $\mathrm{p} 49-56$.

Pavesic, M G, 1971, The archaeology of Hells Canyon Creek Rockshelter, Wallowa County, Oregon: unpub PhD thesis, Univ Colorado, Boulder; Univ Microfilms, Ann Arbor, Michigan.

Richmond, J M, 1965, Glaciation of the Rocky Mountains, in: Wright, H E and Frey, D G (eds), The Quaternary of United States, Princeton, New Jersey, Princeton Univ Press, p 217.

Scharpenseel, H W, 1972, Messung der Naturlichen C-14 Konzentration in der Organischen Substanz von Rezenten Böden, Eine Swischenbilanz: A Pfanz, Bondenkinde, v 133, p 241 . 